\title{
EDITORIAL
}

\section{Towards prognostic biomarkers in pulmonary arterial hypertension}

\author{
J-L. Cracowski
}

$\mathbf{T}$ he most reliable way to assess the clinical impact of a therapeutic intervention in pulmonary arterial hypertension $(\mathrm{PAH})$ is through its effect on well-defined clinical end-points, such as mortality. However, this standard is often impractical because of the long period and large number of patients required to reach these clinical end-points, and substitutes, such as the 6-min walking distance, are often used. Biomarkers may also be reliable substitutes for clinical end-points, and are called surrogate end-points when they meet such requirements. According to the US National Institutes of Health Biomarkers Definitions Working Group, a biomarker is "a characteristic that is objectively measured and evaluated as an indicator of normal biological processes, pathogenic processes, or pharmacologic responses to a therapeutic intervention" [1]. However, while this definition is used for drug discovery, another narrower definition is still commonly used: "disease-associated molecular changes in body tissue and fluids", i.e. a biological marker [2]. Among the estimated 150,000 papers documenting thousands of biological markers, less than 100 biomarkers are routinely used in the clinic [2]. In the field of PAH, which is a rare disease, most biomarker studies examined less than 100 patients, and therefore lacked the statistical power needed to demonstrate a strong association between any biomarker and death. Registries such as the Registry to Evaluate Early And Longterm PAH Disease Management (REVEAL) [3] have the advantage of enabling the study of larger populations and, therefore, have much better statistical power. However, in most cases, registries are not designed to evaluate new biomarkers, as they do not reach the necessary quality of biological sampling with storage of samples in a biobank, and are often a mix of incident and prevalent patients. In addition, many studies have focused on just one biomarker, while there is increasing evidence that a single isolated biomarker will not provide a substantial improvement in risk classification and that a multiple-biomarker approach is required. Indeed, recent multiplex assays, such as those used by Soon et al. [4] for inflammatory cytokine measurement, enable a large number of substances to be readily quantified simultaneously, and we need to determine how to handle and interpret this large quantity of biological information. Furthermore, there is a

Clinical Pharmacology Unit, Inserm CIC03, Grenoble University Hospital, and Inserm U1042, Joseph Fourier University, Grenoble, France.

CORRESPONDENCE: J-L. Cracowski, Clinical Pharmacology Unit, Inserm CIC03, Centre d'Investigation Clinique de Grenoble, CHU de Grenoble, 38043 Grenoble Cedex 09, France. E-mail: Jean-Luc.Cracowski@ujf-grenoble.fr time-lag between preliminary studies showing an independent association with death and the routine use of a biomarker for patients. Before a biomarker can be used routinely, several criteria must be met $[5,6]$. First, the measure should add independent information on PAH prognosis, as shown in a survival analysis based on biomarkers with clinical and haemodynamic parameters as covariates. Secondly, the measure should account for a large proportion of risk. This means that the risk factor frequency must be sufficiently high (e.g. a biomarker such as cardiac troponin $\mathrm{T}(\mathrm{cTnT})$ will provide prognostic information only for the few patients in which it is detectable, although this can be improved using highsensitivity techniques), and that the relative risk should also be sufficiently elevated. There is no cut-off point to quantify the proportion of risk, but a hazard ratio $>2$ is frequently suggested [5, 6]. However, risk usually increases progressively with increasing biomarker levels, so various cut-off points must be evaluated. In most cases, these cut-offs will not represent a specific threshold effect, but a compromise between the proportion of the population at risk and the relative risk of morbidity associated with values higher than the cut-off point. As an example, BenZA et al. [3] clearly showed that brain natriuretic peptide levels $>180 \mathrm{pg} \cdot \mathrm{mL}^{-1}$ were associated with a poor prognosis, with a hazard ratio close to 2 , and the opposite for those with levels $<50 \mathrm{pg} \cdot \mathrm{mL}^{-1}$. The question remains as to whether such an extreme cut-off would be of any help in the clinical decision making process given the fact that most patients will be between these values. Lastly, the test should be reproducible and widely available. While a large number of biomarkers are initially discovered in research laboratories, they need to be quantified rapidly at a low cost for routine use. To date, only a few biomarkers meet these requirements in $\mathrm{PAH}$.

The pathophysiological process of PAH is characterised by endothelial dysfunction of the pulmonary arteries, associated with inflammation and oxidative stress. Vascular remodelling and a proliferation of small pulmonary arteries lead to a chronic elevation of pulmonary vascular resistance, right heart failure and death. The conventional candidate biomarker approach relies on pathophysiology and differentiates between biomarkers of increased right ventricular pre-load, biomarkers of inflammation and oxidative stress, and biomarkers of endothelial dysfunction.

Serum troponin $\mathrm{T}$ is a biomarker of myocardial damage that has been shown to be detectable in $\sim 13 \%$ of group $1 \mathrm{PAH}$ patients [7, 8]. Given its association with right ventricular 
dysfunction, it is a biomarker of increased right ventricular pre-load. In terms of prognosis, positive troponin $\mathrm{T}$ was associated with an increased hazard ratio for death in a heterogeneous group of 56 patients (51 PAH patients), in a multivariate Cox analysis including 6-min walking distance and pulmonary vascular resistance (HR 4.89, 95\% CI $1.18-$ 20.39) [7]. This was further confirmed in a heterogeneous group of pulmonary hypertension patients, but subgroup analysis of 36 patients with idiopathic PAH did not have enough power to demonstrate an association (HR 7.01, 95\% CI 0.68-71). In this issue of the European Respiratory Journal, HERESI et al. [9] tested whether cardiac troponin I provides the same information. This question is important given the fact that in clinical practice, cardiac troponins $\mathrm{T}$ and $\mathrm{I}$ are used interchangeably in coronary artery disease management. However, they differ from a biochemical and analytical point of view, and the equivalence of their performance is debated in conditions such as end-stage renal disease [10]. In addition, it has become clear that even small troponin elevations are associated with adverse health outcomes in coronary artery disease. As a result, there have been several generations of troponin assays, all aimed at tests that reliably detect lower concentrations [11, 12]. Accordingly, using a novel, highsensitivity cTnT assay with a lower detection limit of $2 \mathrm{pg} \cdot \mathrm{mL}^{-1}$, FILUSCH et al. [12] recently showed that concentrations higher than the 99th percentile were observed in $27.3 \%$, compared with $10.9 \%$, of a heterogeneous group of patients with pulmonary hypertension, using the fourth-generation assay. Positive high-sensitivity cTnT was associated with death and was related to right ventricular dysfunction. Using a sensitive cardiac troponin I assay, HerEsI et al. [9] detected cardiac troponin I in $25 \%$ of a group of patients with group 1 $\mathrm{PAH}$, compared with $6.5 \%$ for a classical cTnT assay. All patients with detectable cTnT had detectable cardiac troponin I. Detectable cardiac troponin I was associated with a hazard ratio of 4.74 (95\% CI 1.89-11.89) for death related to right ventricular failure or transplant, and a hazard ratio of $3.2(95 \%$ CI 1.40-7.37) for all-cause mortality and death. There is reasonable evidence to assume that $\mathrm{PAH}$ patients with detectable serum troponin, whether $\mathrm{T}$ or I, are at increased risk of death, and that using more sensitive assays will help us to identify more patients at risk.

There is increasing evidence that inflammation and oxidative stress play a role in the pathophysiology of PAH. Plasma cytokines, including interleukin (IL)- $1 \alpha$, IL-2, IL-4, IL-6, IL-8, IL-10, IL-12 p70, tumour necrosis factor- $\beta$, monocyte chemotactic protein-1 and osteopontin, were found to be elevated in patients with group $1 \mathrm{PAH}[4,13-15]$. Growth differentiation factor-15, a stress responsive member of the transforming growth factor- $\beta$ superfamily, was above the reference limit in $55 \%$ of 76 group $1 \mathrm{PAH}$ patients. C-reactive protein (CRP) was similarly increased [16], a one-log increase being associated with a hazard ratio for death of 3-4. In contrast, HERESI et al. [9] failed to find any association of high-sensitivity CRP with morbidity and mortality. The two studies used two different assays and the levels in the patients in the study by HERESI et al. [9] were more than twice as high as those in the study by QUARCK et al. [16]. However, this shows that unless studies on large cohorts are performed, the repetition of small cohort studies is mandatory before the routine use of a biomarker.
Moreover, it stresses the fact that biomarker research in $\mathrm{PAH}$ should operate as a large collaborative network, with shared biobanks to enable quicker identification of candidate prognostic biomarkers with better reliability.

Finally, the use of proteomics seems intuitively interesting. While surface-enhanced laser desorption/ionisation time-offlight (SELDI-TOF) mass spectrometry (MS) alone is suitable for analysis of readily obtainable clinical samples, such as plasma and urine, and provides a simple, low-resolution pattern generated from proteins retained on a specific chromatographic surface, in most instances, it does not allow the direct identification of those proteins that may be potential disease biomarkers. ABDUL-SALAM et al. [17] have shown that putative biomarkers characteristic of $\mathrm{PAH}$ can be identified using a conjoint SELDI-TOF MS-proteomics approach, but there is still no clinical application of this. A more specific proteomic method can be used with small cohorts, using cells or tissues samples. Using the global proteomic approach of two-dimensional difference gel electrophoresis/MS to examine transformed lymphocytes from a single family with familial $\mathrm{PAH}$, protein expression patterns distinguished patients with familial PAH from both obligate carriers and married-in control subjects [18]. Nevertheless, at present, while the proteomics approach seems promising for identifying new biomarkers in PAH, we are still far from it being of prognostic interest in clinical practice.

\section{STATEMENT OF INTEREST}

A statement of interest for J-L. Cracowski can be found at www.erj. ersjournals.com/site/misc/statements.xhtml

\section{REFERENCES}

1 Biomarkers Definitions Working Group. Biomarkers and surrogate endpoints: preferred definitions and conceptual framework. Clin Pharmacol Ther 2001; 69: 89-95.

2 Poste G. Bring on the biomarkers. Nature 2011; 469: 156-157.

3 Benza RL, Miller DP, Gomberg-Maitland M, et al. Predicting survival in pulmonary arterial hypertension: insights from the registry to evaluate early and long-term pulmonary arterial hypertension disease management (REVEAL). Circulation 2011; 122: $164-172$.

4 Soon E, Holmes AM, Treacy CM, et al. Elevated levels of inflammatory cytokines predict survival in idiopathic and familial pulmonary arterial hypertension. Circulation 2010; 122: 920-927.

5 Manolio T. Novel risk markers and clinical practice. N Engl J Med 2003; 349: 1587-1589.

6 Ware JH. The limitations of risk factors as prognostic tools. N Engl J Med 2006; 355: 2615-2617.

7 Torbicki A, Kurzyna M, Kuca P, et al. Detectable serum cardiac troponin $\mathrm{T}$ as a marker of poor prognosis among patients with chronic precapillary pulmonary hypertension. Circulation 2003; 108: 844-848.

8 Fijalkowska A, Kurzyna M, Torbicki A, et al. Serum N-terminal brain natriuretic peptide as a prognostic parameter in patients with pulmonary hypertension. Chest 2006; 129: 1313-1321.

9 Heresi GA, Tang WHW, Aytekin M, et al. Sensitive cardiac troponin I predicts outcomes in pulmonary arterial hypertension. Eur Respir J 2012; 39: 939-944.

10 Giannitsis E, Katus HA. Comparison of cardiac troponin T and troponin I assays - implications of analytical and biochemical differences on clinical performance. Clin Lab 2004; 50: 521-528. 
11 Christenson RH, Phillips D. Sensitive and high sensitivity next generation cardiac troponin assays: more than just a name. Pathology 2011; 43: 213-219.

12 Filusch A, Giannitsis E, Katus HA, et al. High-sensitive troponin T: a novel biomarker for prognosis and disease severity in patients with pulmonary arterial hypertension. Clin Sci (Lond) 2010; 119: 207-213.

13 Humbert M, Monti G, Brenot F, et al. Increased interleukin-1 and interleukin-6 serum concentrations in severe primary pulmonary hypertension. Am J Respir Crit Care Med 1995; 151: 1628-1631.

14 Itoh $\mathrm{T}$, Nagaya $\mathrm{N}$, Ishibashi-Ueda $\mathrm{H}$, et al. Increased plasma monocyte chemoattractant protein-1 level in idiopathic pulmonary arterial hypertension. Respirology 2006; 11: 158-163.
15 Lorenzen JM, Nickel N, Kramer R, et al. Osteopontin in patients with idiopathic pulmonary hypertension. Chest 2011; 139: 1010-1017.

16 Quarck R, Nawrot T, Meyns B, et al. C-reactive protein: a new predictor of adverse outcome in pulmonary arterial hypertension. J Am Coll Cardiol 2009; 53: 1211-1218.

17 Abdul-Salam VB, Paul GA, Ali JO, et al. Identification of plasma protein biomarkers associated with idiopathic pulmonary arterial hypertension. Proteomics 2006; 6: 2286-2294.

18 Meyrick BO, Friedman DB, Billheimer DD, et al. Proteomics of transformed lymphocytes from a family with familial pulmonary arterial hypertension. Am J Respir Crit Care Med 2008; 177: 99-107. 